\title{
Model Predictive Control for Continuous-Time Singular Jump Systems with Incomplete Transition Rates
}

\author{
Xinxin Gu, Jiwei Wen, and Li Peng \\ Key Laboratory for Advanced Process Control of Light Industry of the Ministry of Education, \\ School of Internet of Things Engineering, Jiangnan University, Wuxi 214122, China \\ Correspondence should be addressed to Jiwei Wen; wjw8143@aliyun.com
}

Received 4 May 2015; Revised 12 July 2015; Accepted 21 July 2015

Academic Editor: Petko Petkov

Copyright (C) 2015 Xinxin Gu et al. This is an open access article distributed under the Creative Commons Attribution License, which permits unrestricted use, distribution, and reproduction in any medium, provided the original work is properly cited.

This paper is concerned with model predictive control (MPC) problem for continuous-time Markov Jump Systems (MJSs) with incomplete transition rates and singular character. Sufficient conditions for the existence of a model predictive controller, which could optimize a quadratic cost function and guarantee that the system is piecewise regular, impulse-free, and mean square stable, are given in two cases at each sampling time. Since the MPC strategy is aggregated into continuous-time singular MJSs, a discretetime controller is employed to deal with a continuous-time plant and the cost function not only refers to the singularity but also considers the sampling period. Moreover, the feasibility of the MPC scheme and the mean square admissibility of the closed-loop system are deeply discussed by using the invariant ellipsoid. Finally, a numerical example is given to illustrate the main results.

\section{Introduction}

As a class of stochastic hybrid systems, Markov Jump Systems (MJSs) are suitable to describe many practical systems whose structures and parameters suffer from random abrupt changes [1-5]. Furthermore, MJSs, which contain a non-full rank system matrix, are usually referred to as singular MJSs, and they have obtained much attention from mathematical and control community. It should be mentioned that a singular MJS can better describe some physical systems than standard MJS model and has extensive applications, such as microelectronic circuits and economics [6-9].

In recent years, an ever increasing number of scholars have paid much attention in the study of singular MJSs $[6,10-12]$. In [13], Boukas investigated the state feedback control for singular MJSs with norm-bounded uncertainties. By considering the full and partial knowledge of transition rates, sufficient conditions which guarantee that the closedloop system is piecewise regular, impulse-free, and mean square stable are given in [14]. Due to the regularity and pulse phenomenon, the stability and stabilizability of singular MJSs are intensively studied [10]. In [11], the authors aimed to study the mean square stability of singular MJSs and extended the main criterion to frequency domain. The robust mean square stability problem for uncertain singular MJSs with actuator saturation is further considered [15], in which sufficient conditions for such systems to be regular, causal, and mean square stable are derived in LMI form.

On another research frontline, model predictive control (MPC), which is also named as receding horizon control, has received tremendous attention in practical applications [1619]. At each time instant, MPC requires the online solution of an optimization problem and then gets the optimized control inputs. We calculate a control sequence, but only the first element is implemented. At the next sampling time, we resolve the optimization problem again. Therefore, the control gain is updated at each sampling time and can naturally compensate the model uncertainties and exogenous disturbance to some extent. MPC has been successfully employed in industry since it can cope with hard constraints, control moves, system states, and outputs. As MJS is considered, MPC strategy has also received considerable research efforts on its analysis and synthesis from a theoretical point of view [20-23].

Almost all of the above-mentioned studies are based on the assumption that the transition probabilities are completely known. However, in practice, obtaining the complete 
knowledge of transition probabilities is difficult and costly. Until very recently, some efforts start to focus on MJSs with incomplete transition probabilities. Zhang and Boukas firstly proposed an analysis and design method for MJSs with partly unknown transition probability, which does not require the information of unknown elements [24]. Subsequently, necessary and sufficient conditions for the stability analysis of MJSs with incomplete transition descriptions are further given in [25]. Based on the results of [24, 25], Zheng and Yang [26] investigated the robust stabilization problem with a sliding mode control approach. Shen et al. proposed a new method for the $H_{\infty}$ state feedback control [27] and established sufficient conditions for the existence of the $H_{\infty}$ static output feedback controller [28].

It is worth noting that MPC for MJSs are usually based on discrete-time version. However, in the real world, we take the controlled objects as continuous-time models. To the best of our knowledge, few results about continuous-time singular MJSs are addressed due to the difficulty in stability analysis and controller design, especially further considering incomplete transition rates. In this paper, a new MPC method is developed for continuous-time singular MJSs with incomplete transitions rates. Our main contributions include the following three aspects. (1) A sampled-period MPC law is introduced to deal with the continuous-time plant. (2) A modified Lyapunov function approach is employed to deal with analysis and design problems for the underlying system concerning incomplete information. The approach not only refers to the knowledge of system state but also considers the sampling period and the singularity. (3) The feasibility of the MPC scheme for the continuous-time singular MJS and the mean square admissibility of the closed-loop system are discussed by using invariant ellipsoid.

\section{Problem Statement}

Consider a continuous-time singular MJS with uncertainties described as follows:

$$
\begin{aligned}
E \dot{x}(t)= & (A(r(t))+\Delta A(r(t))) x(t) \\
& +(B(r(t))+\Delta B(r(t))) u(t),
\end{aligned}
$$

where $x(t) \in \mathbb{R}^{n}$ is the system state, $u(t) \in \mathbb{R}^{m}$ is the control input, $E$ is a real constant matrix with $\operatorname{rank}(E)=r(r<$ $n), A(r(t))$ and $B(r(t))$ are known mode-dependent constant matrices with appropriate dimensions, and $\Delta A(r(t))$ and $\Delta B(r(t))$ are matrices with unknown uncertainties satisfying

$$
\begin{aligned}
& \Delta A(r(t))=D F(r(t), t) H_{1}, \\
& \Delta B(r(t))=D F(r(t), t) H_{2},
\end{aligned}
$$

where $D \in \mathbb{R}^{n \times p}, H_{1} \in \mathbb{R}^{q \times n}$, and $H_{2} \in \mathbb{R}^{q \times m}$ are known constant matrices and $F(r(t), t) \in \mathbb{R}^{p \times q}$ is a time-varying matrix function satisfying $F^{T}(r(t), t) F(r(t), t) \leq I .\{r(t), t \geq$ $0\}$ is a continuous-time Markov process which takes values in a finite set $\mathbb{M}=\{1,2, \ldots, N\}$ with the following transition rate:

$$
\begin{aligned}
& P_{r}\{r(t+h)=j \mid r(t)=i\} \\
& \quad= \begin{cases}\lambda_{i j} h+o(h), & i \neq j \\
1+\lambda_{i j} h+o(h), & i=j,\end{cases} \\
& \lambda_{i i}=-\sum_{j \in \mathbb{M}, i \neq j} \lambda_{i j}, \quad \lambda_{i j} \geq 0,
\end{aligned}
$$

where $h>0$ and $\lim _{h \rightarrow 0} o(h) / h \rightarrow 0$ and $\lambda_{i j}$ is the switching rate from mode $i$ at time $t$ to mode $j$ at time $t+h$.

For the sake of convenience, $A(r(t)), B(r(t)), \Delta A(r(t))$, and $\Delta B(r(t))$ could be denoted by $A_{i}, B_{i}, \Delta A_{i}$, and $\Delta B_{i}$, respectively, where $r(t)=i \in \mathbb{M}$.

Moreover, in this paper, the transition rates are considered to be partly available; namely, some elements in matrix $\Lambda$ are unknown. Taking singular MJS (1) with four operation modes as an example, the transition rate matrix could be expressed as

$$
\widetilde{\Lambda}=\left[\begin{array}{llll}
\lambda_{11} & \lambda_{12} & \hat{\lambda}_{13} & \hat{\lambda}_{14} \\
\hat{\lambda}_{21} & \hat{\lambda}_{22} & \lambda_{23} & \lambda_{24} \\
\lambda_{31} & \hat{\lambda}_{32} & \lambda_{33} & \hat{\lambda}_{34} \\
\hat{\lambda}_{41} & \lambda_{42} & \hat{\lambda}_{43} & \lambda_{44}
\end{array}\right],
$$

where the elements with “へ” represent the unknown transition rate.

In this paper, we denote $\mathbb{M}=\mathbb{M}_{k}^{i}+\mathbb{M}_{u k}^{i}, \forall i \in \mathbb{M}$, with

$$
\begin{gathered}
\mathbb{M}_{k}^{i} \triangleq\left\{j: \lambda_{i j} \text { is known }\right\}, \\
\mathbb{M}_{u k}^{i} \triangleq\left\{j: \lambda_{i j} \text { is unknown }\right\} .
\end{gathered}
$$

In addition, if $\mathbb{M}_{k}^{i} \neq \phi$, it can be written as

$$
\mathbb{M}_{k}^{i}=\left\{K_{1}^{i}, \ldots, K_{m}^{i}\right\}, \quad \forall 1 \leq m \leq N,
$$

where $K_{m}^{i} \in \mathbb{N}^{+}$represent the $m$ th known element in the $i$ th row of matrix $\Lambda$. Also, we denote $\lambda_{k}^{i} \triangleq \sum_{j \in \mathbb{M}_{k}^{i}} \lambda_{i j}$.

In the case that $\hat{\lambda}_{i i}$ is unknown, providing a lower bound $\lambda_{d}^{i}$ with $\lambda_{d}^{i} \leq-\lambda_{k}^{i}$ is necessary.

MPC for (1) is studied over an infinite horizon. At each sampling time $k T$ for $k=0,1, \ldots$, we could obtain control gains by making use of the LMI techniques and we will only implement the first calculated control input $u(k T)=F_{i} x(k T)$, where $T$ represents the fixed sampling interval. At the next sampling time, we will calculate the state $x((k+1) T)$ and then compute the feedback control gain $F_{i}$ once again. Let $x(k T, k T)$ denote the state measured at sampling time $k T$; the predicted state at time $k T+\tau$ is denoted by $x(k T+\tau, k T)$; $u(k T+\tau, k T)$ is the control action at time $k T+\tau . x(k T, k T)$ can be noted as $x(k T)$ for simplicity.

About the singular MJS (1), we give the following definitions. 
Definition 1 (see [10]). The continuous-time singular MJSs (1) are said to be

(1) regular if $\operatorname{det}\left(s E-A_{i}\right)$ is not identically zero for each $i \in \mathbb{M}$;

(2) impulse-free if $\operatorname{deg}\left(\operatorname{det}\left(s E-A_{i}\right)\right)=\operatorname{rank}(E)$ for each $i \in \mathbb{M}$;

(3) mean square stable if there exists a scalar $M\left(x_{0}, r_{0}\right)>$ 0 , for any $x_{0} \in \mathbb{R}^{n}, r_{0} \in \mathbb{M}$, such that

$$
\mathbf{E}\left\{\int_{0}^{\infty}\|x(t)\|^{2} d t \mid x_{0}, r_{0}\right\} \leq M\left(x_{0}, r_{0}\right)
$$

(4) mean square admissible if it is regular, impulse-free, and mean square stable.

Definition 2 (see [29] (invariant ellipsoid)). Given a continuous-time dynamical system $\dot{x}(t)=f(x(t))$, a subset $\mathbb{E}=\left\{x \in R^{n} \mid x^{T} Q^{-1} x \leq 1\right\}$ of the state space $\mathbb{R}^{n}$ is said to be an invariant ellipsoid, if it has the property that whenever $x(k T) \in \mathbb{E}$, then $x(N T) \in \mathbb{E}$ for all times $N T>k T$.

Before giving the main results of the singular MJS (1), some useful lemmas are presented below.

Lemma 3 (see [30]). Given matrices $G_{1}$ and $G_{2}$ with appropriate dimensions and a symmetric matrix $\Omega, \Omega+G_{1} F(t) G_{2}+$ $G_{2}{ }^{T} F(t)^{T} G_{1}{ }^{T}<0$, for all $F(t)^{T} F(t) \leq I$, if and only if there exists a scalar $\delta>0$ such that

$$
\Omega+\delta G_{1} G_{1}^{T}+\frac{1}{\delta} G_{2}^{T} G_{2}<0 .
$$

Lemma 4 (see [31]). Given symmetric and positive definite matrices $M$ and $N$, if $M \geq N>0$, then $N^{-1} \geq M^{-1}>0$.

Lemma 5 (see [14]). If a mode-dependent symmetric matrix $P_{i}$ and a real constant matrix $E$ with $\operatorname{rank}(E)=r(r<n)$ are given a priori, then we will have $E^{T} P_{i} \leq(1 / 4) \widetilde{\varepsilon}_{i}^{-1} I+\widetilde{\varepsilon}_{i} E^{T} P_{i} P_{i}^{T} E$ for any $\varepsilon_{i}>0$, where I presents the identity matrices with compatible dimensions.

In this paper, at each sampling time $k T$, we aim to design a state feedback controller

$$
u(k T+\tau, k T)=F_{i} x(k T+\tau, k T) \quad \tau \geq 0,
$$

by solving the following optimization problem:

$$
\begin{aligned}
& \min _{F_{i}} J_{k}, \\
& J_{k}:=\int_{0}^{\infty}\left(x^{T}(k T+\tau, k T) Q_{c} x(k T+\tau, k T)\right. \\
& \left.\quad+u^{T}(k T+\tau, k T) R u(k T+\tau, k T)\right) d \tau,
\end{aligned}
$$

where $Q_{c}>0$ and $R>0$ are symmetric weighting matrices, $J_{k}$ is the performance index of system (1) at time $k T$, and $F_{i}$ is the state feedback control gain.

\section{Model Predictive Controller Design}

In this section, we will design MPC controller for the singular MJSs with incomplete transition rates by presenting sufficient conditions, which could be efficiently solved by LMI toolbox.

Theorem 6. Consider the singular MJS (1) with incomplete transition rates and let $x(k T)$ be the state of uncertain singular system (1) at sampling time $k T$. One will obtain $Q_{i}$ and $Y_{i}$ by solving the following optimization problem:

$$
\min _{\gamma, Q_{i}, Y_{i}, \varepsilon_{i}, \delta_{i}} \gamma
$$

satisfying

$$
\left[\begin{array}{ccc}
1 & x^{T}(k T) & x^{T}(k T) E^{T} \\
* & 4 \varepsilon_{i} I & 0 \\
* & * & Q_{i}+Q_{i}^{T}-\varepsilon_{i} I
\end{array}\right] \geq 0 \quad i \in \mathbb{M},
$$

$$
\left[\begin{array}{cccccccc}
\Omega_{1} & Q_{i}^{T} & Y_{i}^{T} & \left(H_{1} Q_{i}+H_{2} Y_{i}\right)^{T} & \Omega_{2} & \Omega_{4} & \sqrt{-\lambda_{k}^{i}} Q_{i}^{T} & \sqrt{-\lambda_{k}^{i}} Q_{i}^{T} E^{T} \\
* & -\gamma Q_{c}^{-1} & 0 & 0 & 0 & 0 & 0 & 0 \\
* & * & -\gamma R^{-1} & 0 & 0 & 0 & 0 & 0 \\
* & * & * & -\delta_{i} & 0 & 0 & 0 & 0 \\
* & * & * & * & -\Omega_{3} & 0 & 0 & 0 \\
* & * & * & * & * & -\Omega_{5} & 0 & 0 \\
* & * & * & * & * & * & -4 \varepsilon_{j} I & 0 \\
* & * & * & * & * & * & * & -Q_{j}-Q_{j}^{T}+\varepsilon_{j} I
\end{array}\right] \leq 0 \quad \forall j \in \mathbb{M}_{u k}^{i}, \quad \text { if } i \in \mathbb{M}_{k}^{i},
$$




$$
\left[\begin{array}{cccccccc}
\Omega_{1}^{*} & Q_{i}^{T} & Y_{i}^{T} & \left(H_{1} Q_{i}+H_{2} Y_{i}\right)^{T} & \Omega_{2} & \Omega_{4} & \sqrt{-\lambda_{d}^{i}-\lambda_{k}^{i}} Q_{i}^{T} & \sqrt{-\lambda_{d}^{i}-\lambda_{k}^{i}} Q_{i}^{T} E^{T} \\
* & -\gamma Q_{c}^{-1} & 0 & 0 & 0 & 0 & 0 & 0 \\
* & * & -\gamma R^{-1} & 0 & 0 & 0 & 0 & 0 \\
* & * & * & -\delta_{i} & 0 & 0 & 0 & 0 \\
* & * & * & * & -\Omega_{3} & 0 & 0 & 0 \\
* & * & * & * & * & -\Omega_{5} & 0 & 0 \\
* & * & * & * & * & * & -4 \varepsilon_{j} I & 0 \\
* & * & * & * & * & * & * & -Q_{j}-Q_{j}^{T}+\varepsilon_{j} I
\end{array}\right] \leq 0 \quad \forall j \in \mathbb{M}_{u k}^{i}, \text { if } i \in \mathbb{M}_{u k}^{i},
$$

where

$\Omega_{1}$

$$
=A_{i} Q_{i}+B_{i} Y_{i}+\left(A_{i} Q_{i}+B_{i} Y_{i}\right)^{T}+\delta_{i} D D^{T}+\lambda_{i i} Q_{i}^{T} E^{T},
$$

$\Omega_{1}^{*}$

$$
\begin{aligned}
& =A_{i} Q_{i}+B_{i} Y_{i}+\left(A_{i} Q_{i}+B_{i} Y_{i}\right)^{T}+\delta_{i} D D^{T}-\lambda_{k}^{i} Q_{i}^{T} E^{T}, \\
& \Omega_{2}=\left[\begin{array}{lll}
\sqrt{\lambda_{i \mathbb{M}_{1}^{i}}} Q_{i}^{T} & \cdots & \sqrt{\lambda_{i \mathbb{M}_{m}^{i}}} Q_{i}^{T}
\end{array}\right], \\
& \Omega_{3}=\operatorname{diag}\left\{\begin{array}{lll}
4 \varepsilon_{\mathbb{M}_{1}^{i}} I & \cdots & \left.4 \varepsilon_{\mathbb{M}_{m}^{i}} I\right\}
\end{array}\right\}
\end{aligned}
$$

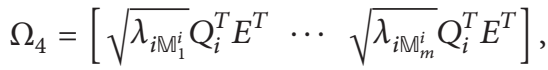

$$
\begin{aligned}
& \Omega_{5} \\
& =\operatorname{diag}\left\{Q_{\mathbb{M}_{1}^{i}}+Q_{\mathbb{M}_{1}^{i}}^{T}-\varepsilon_{\mathbb{M}_{1}^{i}} I \cdots Q_{\mathbb{M}_{m}^{i}}+Q_{\mathbb{M}_{m}^{i}}^{T}-\varepsilon_{\mathbb{M}_{m}^{i}} I\right\} .
\end{aligned}
$$

The mode-dependent state feedback gain $F_{i}$ is given by

$$
F_{i}=Y_{i} Q_{i}^{-1} \text {. }
$$

Proof. Define a quadratic function

$$
\begin{aligned}
V(x(t)) & =x^{T}(t) E^{T} P_{i} x(t), \\
E^{T} P_{i} & =P_{i}^{T} E \geq 0 .
\end{aligned}
$$

Here, we use $\Gamma$ to denote the weak infinitesimal generator of random process $\{x(t), i, t \geq 0\}$ for each mode $i \in \mathbb{M}$; it is defined as

$$
\begin{aligned}
\Gamma & (V(x(t), r(t)=i)) \\
& =\lim _{\delta \rightarrow 0^{+}} \frac{1}{\delta}\{\mathbf{E}[V(x(t+\delta), r(t+\delta)) \mid x(t), r(t) \\
& =i]-V(x(t), i)\} .
\end{aligned}
$$

At each sampling time $k T$, suppose that $V$ satisfy the following inequality:

$$
\begin{aligned}
\Gamma( & V(x(k T+\tau, k T), i)) \\
& \leq-\left(x^{T}(k T+\tau, k T) Q_{c} x(k T+\tau, k T)\right. \\
& \left.+u^{T}(k T+\tau, k T) R u(k T+\tau, k T)\right) .
\end{aligned}
$$

Because the robust cost function will eventually become $x(\propto, k T)=0$, we will get

$$
J_{k} \leq V(x(k T), i)=x^{T}(k T) E^{T} P_{i} x(k T),
$$

by integrating both the left and the right sides of inequality (19) with $\tau \in\left[\begin{array}{ll}0 & \propto\end{array}\right)$.

Considering Lemma 5, for any $\widetilde{\varepsilon}_{i} \geq 0$, we will have

$$
\begin{aligned}
& V(x(k T), i)=x^{T}(k T) E^{T} P_{i} x(k T) \\
& \quad \leq x^{T}(k T)\left(\frac{1}{4} \widetilde{\varepsilon}_{i}^{-1} I+\widetilde{\varepsilon}_{i} E^{T} P_{i} P_{i}^{T} E\right) x(k T) ;
\end{aligned}
$$

let

$$
\begin{aligned}
x^{T}(k T)\left(\frac{1}{4} \widetilde{\varepsilon}_{i}^{-1} I+\widetilde{\varepsilon}_{i} E^{T} P_{i} P_{i}^{T} E\right) x(k T) & \leq \gamma, \\
\widetilde{\varepsilon}_{i} & =\gamma^{-1} \varepsilon_{i}, \\
P_{i} & =\gamma Q_{i}^{-1} ;
\end{aligned}
$$

then, we have

$$
\begin{aligned}
1-x^{T}(k T)\left(\frac{1}{4} \varepsilon_{i}^{-1} I\right) x(k T) \\
-x^{T}(k T)\left(\varepsilon_{i} E^{T} Q_{i}^{-1} Q_{i}^{-T} E\right) x(k T) \geq 0 .
\end{aligned}
$$

Using the fact that $\varepsilon_{i}^{-1} Q_{i} Q_{i}^{T} \geq Q_{i}+Q_{i}^{T}-\varepsilon_{i} I$ for any $\varepsilon_{i}>0$ and Lemma 4 , we will obtain

$$
\varepsilon_{i} Q_{i}^{-1} Q_{i}^{-T} \leq\left(Q_{i}+Q_{i}^{T}-\varepsilon_{i} I\right)^{-1}
$$

Obviously, via the well-known Schur complement, it is easy to get that conditions (12) and (24) imply (23).

Applying the control law (9) and the definition of weak infinitesimal generator, inequality (19) becomes

$$
\begin{aligned}
\left(\left(A_{i}+\Delta A_{i}\right)+\left(B_{i}+\Delta B_{i}\right) F_{i}\right)^{T} P_{i} \\
+P_{i}^{T}\left(\left(A_{i}+\Delta A_{i}\right)+\left(B_{i}+\Delta B_{i}\right) F_{i}\right) \\
+\sum_{j \in \mathbb{M}} \lambda_{i j} E^{T} P_{j}+Q_{c}+F_{i}^{T} R F_{i} \leq 0
\end{aligned}
$$


Pre- and postmultiplying (25) by $P_{i}^{-T}$ and its transpose, replacing $P_{i}$ and $Y_{i}$, by $\gamma Q_{i}^{-1}$ and $F_{i} Q_{i}$, respectively, one will get

$$
\begin{aligned}
\left(A_{i} Q_{i}\right. & \left.+B_{i} Y_{i}\right)+\left(A_{i} Q_{i}+B_{i} Y_{i}\right)^{T}+\Delta A_{i} Q_{i}+\Delta B_{i} Y_{i} \\
& +Q_{i}^{T} \Delta A_{i}^{T}+Y_{i}^{T} \Delta B_{i}^{T}+\gamma^{-1} \sum_{j \in \mathbb{M}} Q_{i}^{T} \lambda_{i j} E^{T} P_{j} Q_{i} \\
& +\gamma^{-1} Q_{i}^{T} Q_{c} Q_{i}+\gamma^{-1} Y_{i}^{T} R Y_{i} \leq 0 ;
\end{aligned}
$$

considering the unknown uncertainty (2), (26) becomes

$$
\begin{aligned}
\left(A_{i} Q_{i}\right. & \left.+B_{i} Y_{i}\right)+\left(A_{i} Q_{i}+B_{i} Y_{i}\right)^{T} \\
& +D F_{i}(t)\left(H_{1} Q_{i}+H_{2} Y_{i}\right) \\
& +\left(H_{1} Q_{i}+H_{2} Y_{i}\right)^{T} F_{i}^{T}(t) D^{T} \\
& +\gamma^{-1} \sum_{j \in \mathbb{M}} Q_{i}^{T} \lambda_{i j} E^{T} P_{j} Q_{i}+\gamma^{-1} Q_{i}^{T} Q_{c} Q_{i} \\
& +\gamma^{-1} Y_{i}^{T} R Y_{i} \leq 0 .
\end{aligned}
$$

According to Lemma 3, (27) holds if and only if there exists $\delta_{i}>0$ satisfying

$$
\begin{aligned}
\left(A_{i} Q_{i}\right. & \left.+B_{i} Y_{i}\right)+\left(A_{i} Q_{i}+B_{i} Y_{i}\right)^{T}+\delta_{i} D D^{T} \\
& +\frac{1}{\delta_{i}}\left(H_{1} Q_{i}+H_{2} Y_{i}\right)^{T}\left(H_{1} Q_{i}+H_{2} Y_{i}\right) \\
& +\gamma^{-1} \sum_{j \in \mathbb{M}} Q_{i}^{T} \lambda_{i j} E^{T} P_{j} Q_{i}+\gamma^{-1} Q_{i}^{T} Q_{c} Q_{i} \\
& +\gamma^{-1} Y_{i}^{T} R Y_{i} \leq 0 .
\end{aligned}
$$

According to Lemma 5, (28) holds if (29) is satisfied for any $\widetilde{\varepsilon}_{j}>0$ satisfying

$$
\begin{aligned}
\left(A_{i} Q_{i}\right. & \left.+B_{i} Y_{i}\right)+\left(A_{i} Q_{i}+B_{i} Y_{i}\right)^{T}+\delta_{i} D D^{T} \\
& +\frac{1}{\delta_{i}}\left(H_{1} Q_{i}+H_{2} Y_{i}\right)^{T}\left(H_{1} Q_{i}+H_{2} Y_{i}\right) \\
& +\gamma^{-1} \sum_{j \in \mathbb{M}} Q_{i}^{T} \lambda_{i j}\left(\frac{1}{4} \widetilde{\varepsilon}_{j}^{-1} I+\widetilde{\varepsilon}_{j} E^{T} P_{j} P_{j}^{T} E\right) Q_{i} \\
& +\gamma^{-1} Q_{i}^{T} Q_{c} Q_{i}+\gamma^{-1} Y_{i}^{T} R Y_{i} \leq 0 .
\end{aligned}
$$

Replacing $P_{j}$ and $\widetilde{\varepsilon}_{j}^{-1}$ by $\gamma Q_{j}^{-1}$ and $\gamma \varepsilon_{j}^{-1}$, respectively, we will have

$$
\begin{aligned}
\left(A_{i} Q_{i}\right. & \left.+B_{i} Y_{i}\right)+\left(A_{i} Q_{i}+B_{i} Y_{i}\right)^{T}+\delta_{i} D D^{T} \\
& +\frac{1}{\delta_{i}}\left(H_{1} Q_{i}+H_{2} Y_{i}\right)^{T}\left(H_{1} Q_{i}+H_{2} Y_{i}\right) \\
& +\sum_{j \in \mathbb{M}} \frac{1}{4} \lambda_{i j} Q_{i}^{T} \varepsilon_{j}^{-1} I Q_{i} \\
& +\sum_{j \in \mathbb{M}} \lambda_{i j} Q_{i}^{T} E^{T}\left(Q_{j}^{-1} Q_{j}^{-T} \varepsilon_{j}\right) E Q_{i}+\gamma^{-1} Q_{i}^{T} Q_{c} Q_{i} \\
& +\gamma^{-1} Y_{i}^{T} R Y_{i} \leq 0 .
\end{aligned}
$$

Subsequently, we take transition rate into account and separate it into two cases, $i \in \mathbb{M}_{k}^{i}$ and $i \in \mathbb{M}_{u k}^{i}$, respectively.

Case $1\left(i \in \mathbb{M}_{k}^{i}\right)$. Considering that $\sum_{j \in \mathbb{M}} \lambda_{i j}=\sum_{j \in \mathbb{M}_{k}^{i}} \lambda_{i j}+$ $\sum_{j \in \mathbb{M}_{u k}^{i}} \lambda_{i j}$, the left side of (30) is rewritten as

$$
\begin{aligned}
\theta_{i} \triangleq & \left(A_{i} Q_{i}+B_{i} Y_{i}\right)+\left(A_{i} Q_{i}+B_{i} Y_{i}\right)^{T}+\delta_{i} D D^{T} \\
& +\frac{1}{\delta_{i}}\left(H_{1} Q_{i}+H_{2} Y_{i}\right)^{T}\left(H_{1} Q_{i}+H_{2} Y_{i}\right) \\
& +\gamma^{-1} Q_{i}^{T} Q_{c} Q_{i}+\gamma^{-1} Y_{i}^{T} R Y_{i}+\alpha_{k}^{i} \\
& +\frac{1}{4} \sum_{j \in \mathbb{M}_{u k}^{i}} \hat{\lambda}_{i j} Q_{i}^{T} \varepsilon_{j}^{-1} I Q_{i}+\beta_{k}^{i} \\
& +\sum_{j \in \mathbb{M}_{u k}^{i}} \hat{\lambda}_{i j} Q_{i}^{T} E^{T}\left(Q_{j}^{-1} Q_{j}^{-T} \varepsilon_{j}\right) E Q_{i}
\end{aligned}
$$

where the elements $\hat{\lambda}_{i j}\left(\forall j \in \mathbb{M}_{u k}^{i}\right)$ are unknown, $\alpha_{k}^{i} \triangleq(1 / 4) \sum_{j \in \mathbb{M}_{k}^{i}} \lambda_{i j} Q_{i}^{T} \varepsilon_{j}^{-1} I Q_{i}$, and $\beta_{k}^{i} \triangleq \sum_{j \in \mathbb{M}_{k}^{i}} \lambda_{i j} Q_{i}^{T} E^{T}$ $\left(Q_{j}^{-1} Q_{j}^{-T} \varepsilon_{j}\right) E Q_{i}$. Noting that, in this case, $\lambda_{i i}$ is known, $\lambda_{k}^{i}=$ $\lambda_{i i}+\sum_{j \in \mathbb{M}_{k}^{i}, i \neq j}<0$, and $\sum_{j \in \mathbb{M}} Q_{i}^{T} \lambda_{i j} E^{T}\left(Q_{j}^{-1}\right) Q_{i}=\lambda_{i i} Q_{i}^{T} E^{T}$, $j=i$, then

$$
\begin{aligned}
\theta_{i}= & \left(A_{i} Q_{i}+B_{i} Y_{i}\right)+\left(A_{i} Q_{i}+B_{i} Y_{i}\right)^{T}+\delta_{i} D D^{T} \\
& +\frac{1}{\delta_{i}}\left(H_{1} Q_{i}+H_{2} Y_{i}\right)^{T}\left(H_{1} Q_{i}+H_{2} Y_{i}\right) \\
& +\gamma^{-1} Q_{i}^{T} Q_{c} Q_{i}+\gamma^{-1} Y_{i}^{T} R Y_{i}+\lambda_{i i} Q_{i}^{T} E^{T}+\alpha_{k}^{i *} \\
& +\frac{1}{4} \sum_{j \in \mathbb{M}_{u k}^{i}} \frac{\hat{\lambda}_{i j}}{-\lambda_{k}^{i}}\left(-\lambda_{k}^{i}\right) Q_{i}^{T} \varepsilon_{j}^{-1} I Q_{i}+\beta_{k}^{i *} \\
& +\sum_{j \in \mathbb{M}_{u k}^{i}} \frac{\widehat{\lambda}_{i j}}{-\lambda_{k}^{i}}\left(-\lambda_{k}^{i}\right) Q_{i}^{T} E^{T}\left(Q_{j}^{-1} Q_{j}^{-T} \varepsilon_{j}\right) E Q_{i},
\end{aligned}
$$

where $\alpha_{k}^{i *}=(1 / 4) \sum_{j \in \mathbb{M}_{k}^{i}, j \neq i} \lambda_{i j} Q_{i}^{T} \varepsilon_{j}^{-1} I Q_{i}$ and $\beta_{k}^{i *}=\sum_{j \in \mathbb{M}_{k}^{i}, j \neq i}$ $\lambda_{i j} Q_{i}^{T} E^{T}\left(Q_{j}^{-1} Q_{j}^{-T} \varepsilon_{j}\right) E Q_{i}$, because of $0 \leq \hat{\lambda}_{i j} /-\lambda_{k}^{i} \leq 1$ and $\sum_{j \in \mathbb{M}_{u k}^{i}} \widehat{\lambda}_{i j} /-\lambda_{k}^{i}=1$; we will obtain that

$$
\begin{aligned}
\theta_{i} & =\sum_{j \in \mathbb{M}_{u k}^{i}} \frac{\widehat{\lambda}_{i j}}{-\lambda_{k}^{i}}\left(\left(A_{i} Q_{i}+B_{i} Y_{i}\right)+\left(A_{i} Q_{i}+B_{i} Y_{i}\right)^{T}\right. \\
& +\delta_{i} D D^{T}+\frac{1}{\delta_{i}}\left(H_{1} Q_{i}+H_{2} Y_{i}\right)^{T}\left(H_{1} Q_{i}+H_{2} Y_{i}\right) \\
& +\gamma^{-1} Q_{i}^{T} Q_{c} Q_{i}+\gamma^{-1} Y_{i}^{T} R Y_{i}+\lambda_{i i} Q_{i}^{T} E^{T}+\alpha_{k}^{i *} \\
& +\left(-\lambda_{k}^{i}\right) Q_{i}^{T} E^{T}\left(Q_{j}^{-1} Q_{j}^{-T} \varepsilon_{j}\right) E Q_{i} \\
& \left.+\frac{1}{4}\left(-\lambda_{k}^{i}\right) Q_{i}^{T} \varepsilon_{j}^{-1} I Q_{i}+\beta_{k}^{i *}\right) .
\end{aligned}
$$


Since $\theta_{i}<0$, we can have the following inequality:

$$
\begin{aligned}
\left(A_{i} Q_{i}\right. & \left.+B_{i} Y_{i}\right)+\left(A_{i} Q_{i}+B_{i} Y_{i}\right)^{T}+\delta_{i} D D^{T} \\
& +\frac{1}{\delta_{i}}\left(H_{1} Q_{i}+H_{2} Y_{i}\right)^{T}\left(H_{1} Q_{i}+H_{2} Y_{i}\right) \\
& +\gamma^{-1} Q_{i}^{T} Q_{c} Q_{i}+\gamma^{-1} Y_{i}^{T} R Y_{i}+\alpha_{k}^{i *} \\
& +\frac{1}{4}\left(-\lambda_{k}^{i}\right) Q_{i}^{T} \varepsilon_{j}^{-1} I Q_{i}+\beta_{k}^{i *} \\
& +\left(-\lambda_{k}^{i}\right) Q_{i}^{T} E^{T}\left(Q_{j}^{-1} Q_{j}^{-T} \varepsilon_{j}\right) E Q_{i}+\lambda_{i i} Q_{i}^{T} E^{T} \\
< & 0 .
\end{aligned}
$$

Using (24), we can easily get (34), if

$$
\begin{aligned}
\left(A_{i} Q_{i}\right. & \left.+B_{i} Y_{i}\right)+\left(A_{i} Q_{i}+B_{i} Y_{i}\right)^{T}+\delta_{i} D D^{T} \\
& +\frac{1}{\delta_{i}}\left(H_{1} Q_{i}+H_{2} Y_{i}\right)^{T}\left(H_{1} Q_{i}+H_{2} Y_{i}\right) \\
& +\lambda_{i i} Q_{i}^{T} E^{T}+\gamma^{-1} Q_{i}^{T} Q_{c} Q_{i}+\gamma^{-1} Y_{i}^{T} R Y_{i}+\alpha_{k}^{i *} \\
& +\sum_{j \in \mathbb{M}_{k}^{i}, j \neq i} \lambda_{i j} Q_{i}^{T} E^{T}\left(Q_{j}+Q_{j}^{T}-\varepsilon_{j}\right)^{-1} E Q_{i} \\
& +\frac{1}{4}\left(-\lambda_{k}^{i}\right) Q_{i}^{T} \varepsilon_{j}^{-1} I Q_{i} \\
& +\left(-\lambda_{k}^{i}\right) Q_{i}^{T} E^{T}\left(Q_{j}+Q_{j}^{T}-\varepsilon_{j} I\right)^{-1} E Q_{i}<0
\end{aligned}
$$

holds. Applying Schur complement to the above inequality, one can obtain (13).

Case $2\left(i \in \mathbb{M}_{u k}^{i}\right)$. Likewise, considering $\sum_{j \in \mathbb{M}} \lambda_{i j}=$ $\sum_{j \in \mathbb{M}_{k}^{i}} \lambda_{i j}+\sum_{j \in \mathbb{M}_{u k}^{i}} \lambda_{i j}$, we rewrite the left side of (30) as

$$
\begin{aligned}
\theta_{i} \triangleq & \left(A_{i} Q_{i}+B_{i} Y_{i}\right)+\left(A_{i} Q_{i}+B_{i} Y_{i}\right)^{T}+\delta_{i} D D^{T} \\
& +\frac{1}{\delta_{i}}\left(H_{1} Q_{i}+H_{2} Y_{i}\right)^{T}\left(H_{1} Q_{i}+H_{2} Y_{i}\right) \\
& +\gamma^{-1} Q_{i}^{T} Q_{c} Q_{i}+\gamma^{-1} Y_{i}^{T} R Y_{i}+\widehat{\lambda}_{i i} Q_{i}^{T} E^{T} \\
& +\frac{1}{4} \sum_{j \in \mathbb{M}_{k}^{i}} \lambda_{i j} Q_{i}^{T} \varepsilon_{j}^{-1} I Q_{i}+\frac{1}{4} \sum_{j \in \mathbb{M}_{u k}^{i}, j \neq i} \widehat{\lambda}_{i j} Q_{i}^{T} \varepsilon_{j}^{-1} I Q_{i} \\
& +\sum_{j \in \mathbb{M}_{k}^{i}} \lambda_{i j} Q_{i}^{T} E^{T}\left(Q_{j}^{-1} Q_{j}^{-T} \varepsilon_{j}\right) E Q_{i} \\
& +\sum_{j \in \mathbb{M}_{u k}^{i}, j \neq i} \hat{\lambda}_{i j} Q_{i}^{T} E^{T}\left(Q_{j}^{-1} Q_{j}^{-T} \varepsilon_{j}\right) E Q_{i} .
\end{aligned}
$$

Noting that, in this case, $\widehat{\lambda}_{i i}$ is unknown, $\lambda_{k}^{i}=-\widehat{\lambda}_{i i}-$ $\sum_{j \in \mathbb{M}_{u k}^{i}, j \neq i} \lambda_{i j}>0$, then

$$
\begin{aligned}
\theta_{i}= & \left(A_{i} Q_{i}+B_{i} Y_{i}\right)+\left(A_{i} Q_{i}+B_{i} Y_{i}\right)^{T}+\delta_{i} D D^{T} \\
& +\frac{1}{\delta_{i}}\left(H_{1} Q_{i}+H_{2} Y_{i}\right)^{T}\left(H_{1} Q_{i}+H_{2} Y_{i}\right) \\
& +\gamma^{-1} Q_{i}^{T} Q_{c} Q_{i}+\gamma^{-1} Y_{i}^{T} R Y_{i}+\alpha_{k}^{i}+\frac{1}{4}\left(-\hat{\lambda}_{i i}-\lambda_{k}^{i}\right)
\end{aligned}
$$

$$
\begin{aligned}
& \cdot \sum_{j \in \mathbb{M}_{u k}^{i}, j \neq i} \frac{\hat{\lambda}_{i j}}{-\widehat{\lambda}_{i i}-\lambda_{k}^{i}} Q_{i}^{T} \varepsilon_{j}^{-1} I Q_{i}+\left(-\widehat{\lambda}_{i i}-\lambda_{k}^{i}\right) \\
& \cdot \sum_{j \in \mathbb{M}_{u k}^{i}, j \neq i} \frac{\hat{\lambda}_{i j}}{-\widehat{\lambda}_{i i}-\lambda_{k}^{i}} Q_{i}^{T} E^{T}\left(Q_{j}^{-1} Q_{j}^{-T} \varepsilon_{j}\right) E Q_{i} \\
& +\widehat{\lambda}_{i i} Q_{i}^{T} E^{T}+\beta_{k}^{i} .
\end{aligned}
$$

Similarly, since we have $0 \leq \hat{\lambda}_{i j} /\left(-\hat{\lambda}_{i i}-\lambda_{k}^{i}\right) \leq 1$ and $\sum_{j \in \mathbb{M}_{u k}^{i}, j \neq i}\left(\widehat{\lambda}_{i j} /\left(-\widehat{\lambda}_{i i}-\lambda_{k}^{i}\right)\right)=1$, we can easily obtain

$$
\begin{aligned}
\theta_{i} & =\sum_{j \in \mathbb{M}_{u k}^{i}, j \neq i} \frac{\hat{\lambda}_{i j}}{-\widehat{\lambda}_{i i}-\lambda_{k}^{i}}\left(\left(A_{i} Q_{i}+B_{i} Y_{i}\right)\right. \\
& +\left(A_{i} Q_{i}+B_{i} Y_{i}\right)^{T}+\delta_{i} D D^{T} \\
& +\frac{1}{\delta_{i}}\left(H_{1} Q_{i}+H_{2} Y_{i}\right)^{T}\left(H_{1} Q_{i}+H_{2} Y_{i}\right) \\
& +\gamma^{-1} Q_{i}^{T} Q_{c} Q_{i}+\gamma^{-1} Y_{i}^{T} R Y_{i}+\widehat{\lambda}_{i i} Q_{i}^{T} E^{T}+\alpha_{k}^{i}+\beta_{k}^{i} \\
& +\left(-\widehat{\lambda}_{i i}-\lambda_{k}^{i}\right) Q_{i}^{T} E^{T}\left(Q_{j}^{-1} Q_{j}^{-T} \varepsilon_{j}\right) E Q_{i} \\
& \left.+\frac{1}{4}\left(-\hat{\lambda}_{i i}-\lambda_{k}^{i}\right) Q_{i}^{T} \varepsilon_{j}^{-1} I Q_{i}\right) .
\end{aligned}
$$

Therefore, $\theta_{i}<0$ is equivalent to $\forall j \in \mathbb{M}_{u k}^{i}, j \neq i$,

$$
\begin{aligned}
\left(A_{i} Q_{i}\right. & \left.+B_{i} Y_{i}\right)+\left(A_{i} Q_{i}+B_{i} Y_{i}\right)^{T}+\delta_{i} D D^{T} \\
& +\frac{1}{\delta_{i}}\left(H_{1} Q_{i}+H_{2} Y_{i}\right)^{T}\left(H_{1} Q_{i}+H_{2} Y_{i}\right) \\
& +\gamma^{-1} Q_{i}^{T} Q_{c} Q_{i}+\gamma^{-1} Y_{i}^{T} R Y_{i}+\widehat{\lambda}_{i i} Q_{i}^{T} E^{T}+\alpha_{k}^{i} \\
& +\frac{1}{4}\left(-\hat{\lambda}_{i i}-\lambda_{k}^{i}\right) Q_{i}^{T} \varepsilon_{j}^{-1} I Q_{i} \\
& +\left(-\widehat{\lambda}_{i i}-\lambda_{k}^{i}\right) Q_{i}^{T} E^{T}\left(Q_{j}^{-1} Q_{j}^{-T} \varepsilon_{j}\right) E Q_{i}+\beta_{k}^{i}<0 .
\end{aligned}
$$

As $\hat{\lambda}_{i i}$ is lower bounded by $\lambda_{d}^{i}$, we know that $\lambda_{d}^{i} \leq \hat{\lambda}_{i i}<-\lambda_{k}^{i}$. Thus, (39) holds if the following inequality holds:

$$
\begin{aligned}
\left(A_{i} Q_{i}\right. & \left.+B_{i} Y_{i}\right)+\left(A_{i} Q_{i}+B_{i} Y_{i}\right)^{T}+\delta_{i} D D^{T} \\
& +\frac{1}{\delta_{i}}\left(H_{1} Q_{i}+H_{2} Y_{i}\right)^{T}\left(H_{1} Q_{i}+H_{2} Y_{i}\right) \\
& +\gamma^{-1} Q_{i}^{T} Q_{c} Q_{i}+\gamma^{-1} Y_{i}^{T} R Y_{i}-\lambda_{k}^{i} Q_{i}^{T} E^{T}+\alpha_{k}^{i} \\
& +\frac{1}{4}\left(-\lambda_{d}^{i}-\lambda_{k}^{i}\right) Q_{i}^{T} \varepsilon_{j}^{-1} I Q_{i} \\
& +\left(-\lambda_{d}^{i}-\lambda_{k}^{i}\right) Q_{i}^{T} E^{T}\left(Q_{j}^{-1} Q_{j}^{-T} \varepsilon_{j}\right) E Q_{i}+\beta_{k}^{i}<0 .
\end{aligned}
$$

Similarly, (40) is implied by (24) and

$$
\begin{aligned}
\left(A_{i} Q_{i}\right. & \left.+B_{i} Y_{i}\right)+\left(A_{i} Q_{i}+B_{i} Y_{i}\right)^{T}+\delta_{i} D D^{T} \\
& +\frac{1}{\delta_{i}}\left(H_{1} Q_{i}+H_{2} Y_{i}\right)^{T}\left(H_{1} Q_{i}+H_{2} Y_{i}\right) \\
& +\gamma^{-1} Q_{i}^{T} Q_{c} Q_{i}+\gamma^{-1} Y_{i}^{T} R Y_{i}-\lambda_{k}^{i} Q_{i}^{T} E^{T}+\alpha_{k}^{i} \\
& +\frac{1}{4}\left(-\lambda_{d}^{i}-\lambda_{k}^{i}\right) Q_{i}^{T} \varepsilon_{j}^{-1} I Q_{i}
\end{aligned}
$$




$$
\begin{aligned}
& +\left(-\lambda_{d}^{i}-\lambda_{k}^{i}\right) Q_{i}^{T} E^{T}\left(Q_{j}+Q_{j}^{T}-\varepsilon_{j} I\right)^{-1} E Q_{i} \\
& +\sum_{j \in \mathbb{M}_{k}^{i}} \lambda_{i j} Q_{i}^{T} E^{T}\left(Q_{j}+Q_{j}^{T}-\varepsilon_{j} I\right)^{-1} E Q_{i}<0 .
\end{aligned}
$$

Applying Schur complement to (41), we can obtain (14).

Certainly, MPC for singular MJSs without incomplete transition descriptions and the normal MJS with incomplete transition descriptions can be viewed as two special cases of singular MJS. Then, we have the following corollaries.

Corollary 7. Consider the singular MJS (1) without incomplete transition rate and let $x(k T)$ be the state of singular system (1) at sampling time $k T$. One will obtain $Q_{i}$ and $Y_{i}$ by solving the following optimization problem:

$$
\min _{\gamma, Q_{i}, Y_{i}, \varepsilon_{i}, \delta_{i}} \gamma
$$

satisfying

$$
\left[\begin{array}{ccc}
1 & x^{T}(k T) & x^{T}(k T) E^{T} \\
* & 4 \varepsilon_{i} I & 0 \\
* & * & Q_{i}+Q_{i}^{T}-\varepsilon_{i} I
\end{array}\right] \geq 0 \quad i \in \mathbb{M},
$$$$
\left.\begin{array}{cccccc}
\Omega_{1} & Q_{i}^{T} & Y_{i} & \left(H_{1} Q_{i}+H_{2} Y_{i}\right)^{T} & \Omega_{2} & \Omega_{4} \\
* & -\gamma Q_{c}^{-1} & 0 & 0 & 0 & 0 \\
* & * & -\gamma R^{-1} & 0 & 0 & 0 \\
* & * & * & -\delta_{i} & 0 & 0 \\
* & * & * & * & -\Omega_{3} & 0 \\
* & * & * & * & * & -\Omega_{5}
\end{array}\right]
$$

where

$$
\begin{aligned}
& \Omega_{1}=A_{i} Q_{i}+B_{i} Y_{i}+\left(A_{i} Q_{i}+B_{i} Y\right)^{T}+\delta_{i} D D^{T}+\lambda_{i i} Q_{i}^{T} E^{T},
\end{aligned}
$$

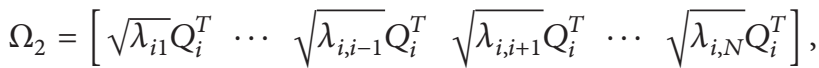

$$
\begin{aligned}
& \Omega_{3}=\operatorname{diag}\left\{\begin{array}{llllll}
4 \varepsilon_{1} I & \cdots & 4 \varepsilon_{i-1} I & 4 \varepsilon_{i+1} I & \cdots & 4 \varepsilon_{N} I
\end{array}\right\} \text {, }
\end{aligned}
$$

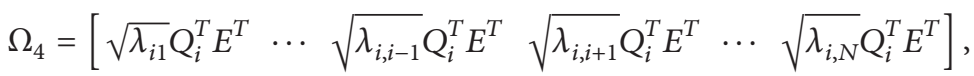

$$
\begin{aligned}
& \Omega_{5}=\operatorname{diag}\left\{Q_{1}+Q_{1}^{T}-\varepsilon_{1} I \cdots Q_{i-1}+Q_{i-1}^{T}-\varepsilon_{i-1} I \quad Q_{i+1}+Q_{i+1}^{T}-\varepsilon_{i+1} I \cdots Q_{N}+Q_{N}^{T}-\varepsilon_{N} I\right\} .
\end{aligned}
$$

Then, the mode-dependent state feedback gain $F_{i}$ can be obtained by (16).

Corollary 8. When $E$ is nonsingular and can be simplified as an identity matrix, system (1) becomes

$$
\begin{aligned}
\dot{x}(t)= & (A(r(t))+\Delta A(r(t))) x(t) \\
& +(B(r(t))+\Delta B(r(t))) u(t) ;
\end{aligned}
$$

let $x(k T)$ be the state of uncertain MJS (45) at sampling time $k T$. One will obtain $Q_{i}$ and $Y_{i}$ by solving the following optimization problem:

$$
\min _{\gamma, Q_{i}, Y_{i}, \delta_{i}} \gamma
$$

satisfying

$\left[\begin{array}{cc}1 & x^{T}(k T) \\ * & Q_{i}\end{array}\right] \geq 0, \quad i \in \mathbb{M}$

$$
\left[\begin{array}{cccccc}
\Omega_{1} & Q_{i}^{T} & Y_{i}^{T} & \left(H_{1} Q_{i}+H_{2} Y_{i}\right)^{T} & \Omega_{2} & \sqrt{-\lambda_{k}^{i}} Q_{i}^{T} \\
* & -\gamma Q_{c}^{-1} & 0 & 0 & 0 & 0 \\
* & * & -\gamma R^{-1} & 0 & 0 & 0 \\
* & * & * & -\delta_{i} & 0 & 0 \\
* & * & * & * & -\Omega_{3} & 0 \\
* & * & * & * & * & -Q_{j}
\end{array}\right] \leq 0
$$

$\forall j \in \mathbb{M}_{u k}^{i}$, if $i \in \mathbb{M}_{k}^{i}$, 
where

$$
\begin{aligned}
& \Omega_{1}=A_{i} Q_{i}+B_{i} Y_{i}+\left(A_{i} Q_{i}+B_{i} Y\right)^{T}+\delta_{i} D D^{T} \\
& +\lambda_{i i} Q_{i}^{T} \\
& \Omega_{1}^{*}=A_{i} Q_{i}+B_{i} Y_{i}+\left(A_{i} Q_{i}+B_{i} Y\right)^{T}+\delta_{i} D D^{T} \\
& +\lambda_{k}^{i} Q_{i}^{T} \\
& \Omega_{2}=\left[\begin{array}{lll}
\sqrt{\lambda_{i \mathbb{M}_{1}^{i}}} Q_{i}^{T} & \cdots & \sqrt{\lambda_{i \mathbb{M}_{m}^{i}}} Q_{i}^{T}
\end{array}\right], \\
& \Omega_{3}=\operatorname{diag}\left\{\begin{array}{lll}
Q_{\mathbb{M}_{1}^{i}} & \cdots & Q_{\mathbb{M}_{m}^{i}}
\end{array}\right\} .
\end{aligned}
$$
(16).

The mode-dependent state feedback gain $F_{i}$ is obtained by

\section{Mean Square Admissibility Analysis}

Previous section gives sufficient conditions, which could guarantee the existence of model predictive controller. In this section, we mainly study the mean square stability of the closed-loop singular MJS (1).

Lemma 9 (feasibility). The solution of the optimization problem in Theorem 6 is feasible over any time interval $[N T,(N+$ 1)T) with $N>k$, if it has the property that the solution is feasible over the time interval $[k T,(k+1) T)$.

Proof. At sampling time $k T$, from inequality (12), we limit the measured state $x(k T)$ in the ellipsoid region $x^{T}\left[(1 / 4) \varepsilon_{i}^{-1} I+\right.$ $\left.E^{T}\left(Q_{i}+Q_{i}^{T}-\varepsilon_{i} I\right)^{-1} E\right] x \leq 1$. According to the proof of Theorem 6 , we can get

$$
\Gamma(V(x(t), r(t)=i))<0 .
$$

Then, for any sampling time NT with $N>k$, the future state $x(N T)$ remains in the ellipsoid set. Therefore, by referring to Definition 2, $x^{T}\left[(1 / 4) \varepsilon_{i}^{-1} I+E^{T}\left(Q_{i}+Q_{i}^{T}-\varepsilon_{i} I\right)^{-1} E\right] x \leq 1$ is an invariant ellipsoid.

The solution of the optimization problem in Theorem 6 is feasible over the time interval $[k T,(k+1) T)$. By observing (12), (13), and (14), it could be concluded that the feasibility of the MPC strategy only depends on (12). Thus, to prove Lemma 3, we only need to prove that the LMI (12) is feasible for all future states $x(N T), N>k$. Considering the invariance of the ellipsoid, we obtain

$$
\begin{gathered}
x^{T}((k+1) T)\left[\frac{1}{4} \varepsilon_{i}^{-1} I+E^{T}\left(Q_{i}+Q_{i}^{T}-\varepsilon_{i} I\right)^{-1} E\right] \\
\cdot x((k+1) T) \leq 1,
\end{gathered}
$$

which means LMI (12) is feasible with state $x((k+1) T)$ and then the feasibility of optimization problem (11) could be achieved over the time interval $[(k+1) T,(k+2) T)$. Similarly, we can complete the proof on the time intervals $[(k+2) T,(k+$ 3)T), $[(k+3) T,(k+4) T), \ldots$

Theorem 10. The feedback predictive control gain computed from Theorem 6 guarantees that the closed-loop singular MJS (1) is mean square admissible.
Proof. Firstly, we show that the singular MJS (1) is regular and impulse-free. There exist two orthogonal matrices $U=$ $\left[\begin{array}{ll}U_{1} & U_{2}\end{array}\right]$ and $V=\left[\begin{array}{ll}V_{1} & V_{2}\end{array}\right]$ such that

$$
\bar{E}=\left[\begin{array}{ll}
I & 0 \\
0 & 0
\end{array}\right]=U^{T} E V^{-T} .
$$

Based on singular value decomposition, system (1) can be written as the following differential and algebraic form:

$$
\begin{aligned}
\dot{x}_{1}(t) & =A_{i c 1}^{*} x_{1}(t)+A_{i c 2}^{*} x_{2}(t), \\
0 & =A_{i c 3}^{*} x_{1}(t)+A_{i c 4}^{*} x_{2}(t),
\end{aligned}
$$

where $A_{i c}=\left(A_{i}+\Delta A_{i}\right)+\left(B_{i}+\Delta B_{i}\right) F_{i}$, and it can be decomposed as $A_{i c}=\left[\begin{array}{ll}A_{i c 1} & A_{i c 2} \\ A_{i c 3} & A_{i c 4}\end{array}\right]$.

Assuming the optimal solution of optimization problem (11) at sampling time $k T$ can be denoted as $P_{i}^{*}(k T)$ and $F_{i}^{*}(k T)$, from the proof of Theorem 6 , we can obtain that there exists $P_{i}^{*}(k T)$ satisfying $(25)$ and

$$
E^{T} P_{i}^{*}(k T)=P_{i}^{T *}(k T) E \geq 0 .
$$

Let

$$
\begin{aligned}
\bar{A}_{i} & =\left[\begin{array}{cc}
A_{i c 1}^{*} & A_{i c 2}^{*} \\
A_{i c 3}^{*} & A_{i c 4}^{*}
\end{array}\right]=U^{T} A_{i c}^{*} V^{-T}, \\
\bar{P}_{i}^{*}(k T) & =\left[\begin{array}{ll}
P_{i 1}^{*}(k T) & P_{i 2}^{*}(k T) \\
P_{i 3}^{*}(k T) & P_{i 4}^{*}(k T)
\end{array}\right]=U^{T} P_{i}^{*}(k T) V^{-T}, \\
\bar{Q}_{c} & =\left[\begin{array}{ll}
Q_{c 1} & Q_{c 2} \\
Q_{c 2}^{T} & Q_{c 3}
\end{array}\right]=V^{-1} Q_{c} V^{-T}, \\
\bar{F}_{i}^{*}(k T) & =\left[\begin{array}{ll}
F_{i 1}^{*}(k T) & \left.F_{i 2}^{*}(k T)\right]=F_{i}^{*}(k T) V^{-T} .
\end{array}\right.
\end{aligned}
$$

By (53), we have $P_{i 1}^{*}(k T)>0$ (because $P_{i}^{*}(k T)$ is nonsingular) and $P_{i 2}^{*}(k T)=0$. Pre- and postmultiplying the left and right sides of (25) by $V^{-1}$ and $V^{-T}$, we obtain

$$
\begin{gathered}
A_{i c 4}^{T *} P_{i 4}^{*}(k T)+P_{i 4}^{T *}(k T) A_{i c 4}^{*}+Q_{c 3} \\
+F_{i 2}^{T *}(k T) R F_{i 2}^{*}(k T)<0 .
\end{gathered}
$$

From (55), we can easily get that $A_{i c 4}^{T *}$ is invertible. Hence, for any $t \geq 0$,

$$
\begin{gathered}
\operatorname{det}\left(s E-A_{i c}^{*}\right)=\operatorname{det} U^{-T} \operatorname{det} V^{T} \operatorname{det}\left(A_{i c 4}^{*}\right) \\
\cdot \operatorname{det}\left(s I-A_{i c 1}^{*}-A_{i c 2}^{*} A_{i c 4}^{(-1) *} A_{i c 3}^{*}\right), \\
\operatorname{deg}\left(\operatorname{det}\left(s E-A_{i c}^{*}\right)\right)=\operatorname{rank}(E),
\end{gathered}
$$

from which we can see that $\operatorname{det}\left(s E-A_{i c}^{*}\right)$ is not identically zero; by Definition 1, the closed-loop system is regular and impulse-free.

What is more, we consider the mean square stability of the singular MJS (1). Here, the piecewise Lyapunov function is described as

$$
\begin{aligned}
V^{*}(x(t), i)=x^{T}(t) E^{T} P_{i}^{*}(k T) x(t), & \\
& t \in[k T,(k+1) T) .
\end{aligned}
$$




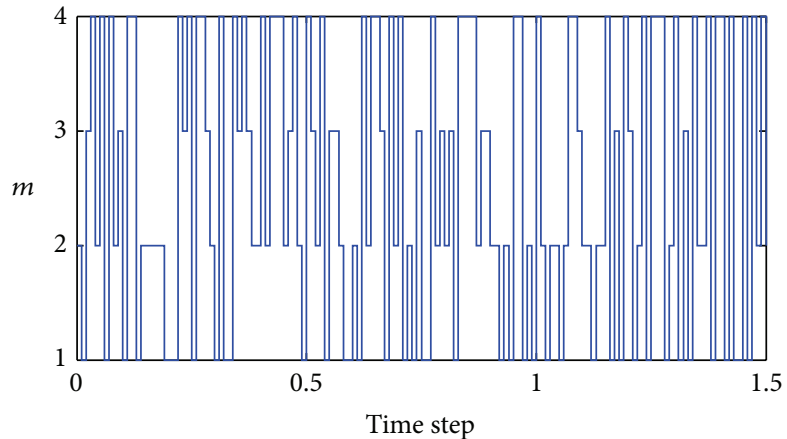

FIGURE 1: Jump mode.

From (25), we have

$$
\begin{aligned}
\left(\left(A_{i}+\Delta A_{i}\right)+\left(B_{i}+\Delta B_{i}\right) F_{i}\right)^{T} P_{i}^{*} \\
+P_{i}^{T *}\left(\left(A_{i}+\Delta A_{i}\right)+\left(B_{i}+\Delta B_{i}\right) F_{i}\right) \\
\quad+\sum_{j \in \mathbb{M}} \lambda_{i j} E^{T} P_{j}^{*} \leq 0
\end{aligned}
$$

which yields

$$
\Gamma\left(V^{*}(x(t), i)\right)<0, \quad t \in[k T,(k+1) T) .
$$

Therefore, $V(x(t), i)$ is strictly decreasing over each time interval $[k T,(k+1) T)$.

Note that $P_{i}^{*}(k T)$ represents a feasible solution at time $(k+$ 1)T while $P_{i}^{*}((k+1) T)$ is an optimal solution at time $(k+1) T$. Since $V^{*}(x(t), i)$ is a piecewise function, we further consider

$$
\begin{aligned}
\lim _{t \rightarrow}(k+1) T^{-} & V^{*}(x(t), i) \\
& =x^{T}((k+1) T) E P_{i}^{*}(k T) x((k+1) T) \\
& \geq x^{T}((k+1) T) E P_{i}^{*}((k+1) T) x((k+1) T) \\
& =V^{*}(x((k+1) T)),
\end{aligned}
$$

for all $k \geq 0$. Inequalities (59) and (60) guarantee that $V(x(t), i)$ is strictly decreasing when $t \in[0, \propto)$; system (1) is mean square stable.

Therefore, according to the property of being regular and impulse-free, the closed-loop singular MJS is mean square admissible.

\section{Numerical Example}

To illustrate the efficiency of the proposed MPC scheme for continuous-time MJSs, a numerical example is presented in the following.

Consider a system with the form of (1), where $r(t)=$ $1,2,3,4$. The system matrices are $A_{1}=\left[\begin{array}{cc}0.75 & -0.75 \\ 0 & -1.5\end{array}\right], A_{2}=$ $\left[\begin{array}{cc}0.15 & -0.49 \\ 0 & -2.1\end{array}\right], A_{3}=\left[\begin{array}{cc}0.3 & -0.15 \\ 0 & -1.8\end{array}\right], A_{4}=\left[\begin{array}{ccc}0.9 & -0.34 \\ 0 & -1.65\end{array}\right], B_{1}=\left[\begin{array}{c}5 \\ -1\end{array}\right]$,

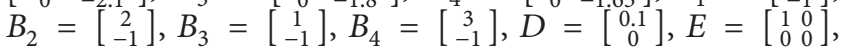
$H_{1}=\left[\begin{array}{ll}0.1 & 0\end{array}\right]$, and $H_{2}=0.1$. The weighting matrices are $Q_{c}=\left[\begin{array}{ll}1 & 0 \\ 0 & 1\end{array}\right]$ and $R=1$.

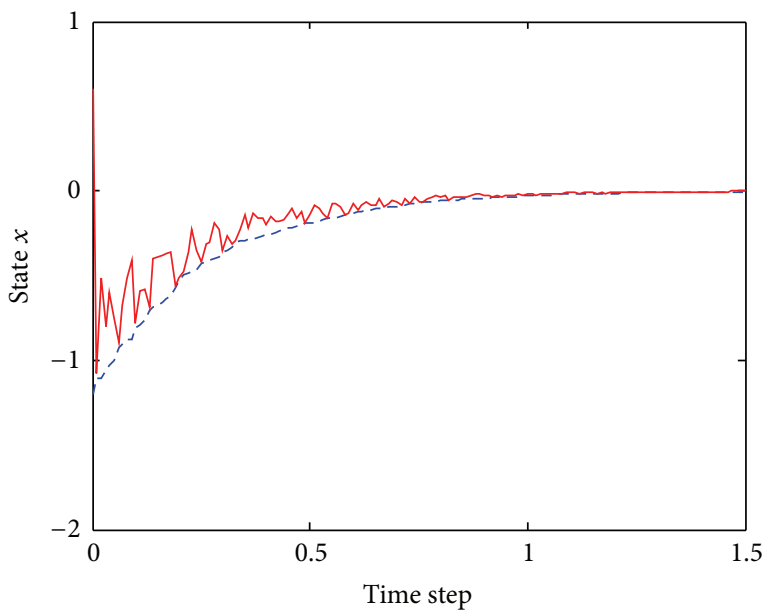

$\begin{array}{rr}--- & x_{1} \\ - & x_{2}\end{array}$

FIGURE 2: State responses under MPC.

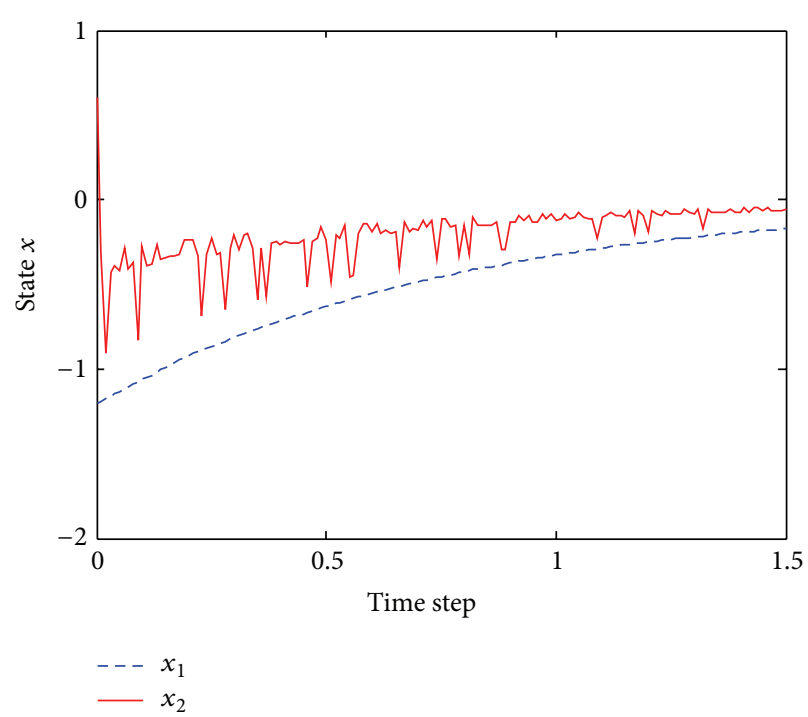

FIGURE 3: State responses under normal state feedback control.

The TRM is given as follows:

$$
\Lambda=\left[\begin{array}{cccc}
-1.3 & 0.2 & \hat{\lambda}_{13} & \hat{\lambda}_{14} \\
\hat{\lambda}_{21} & \hat{\lambda}_{22} & 0.3 & 0.3 \\
0.1 & \hat{\lambda}_{32} & -2.5 & \hat{\lambda}_{34} \\
\hat{\lambda}_{41} & 0.2 & \hat{\lambda}_{43} & -1.2
\end{array}\right],
$$

where the diagonal element $\hat{\lambda}_{22}$ is unknown and its lower bound $\lambda_{d}^{2}=-5$ is a priori given value. The initial value of the state is $x_{0}=\left[\begin{array}{ll}-1.2 & 0.6\end{array}\right]^{T}$ and the initial mode is $r_{0}=1$.

The simulation step is taken as 150 time units and each unit length is taken as 0.01 . We get the jump mode (Figure 1) and apply the designed MPC algorithm and normal state 


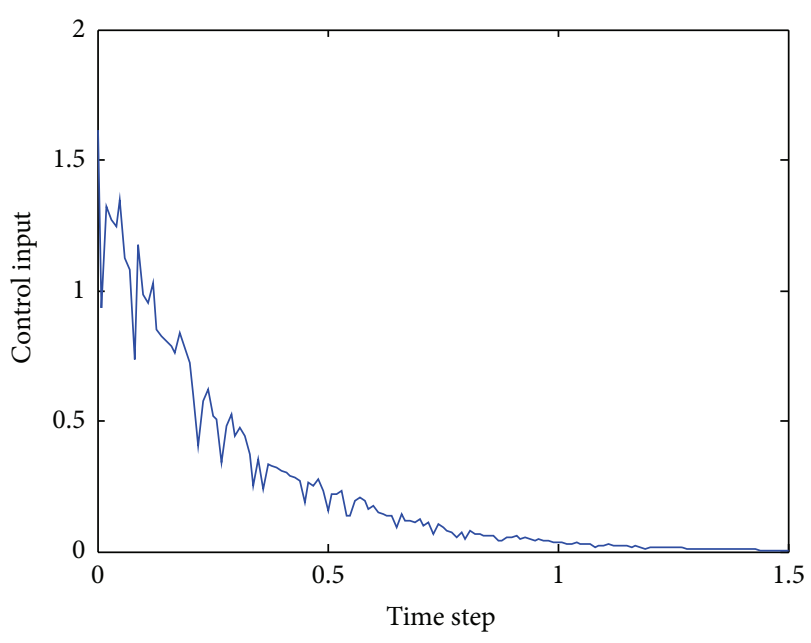

Figure 4: Control input under MPC.

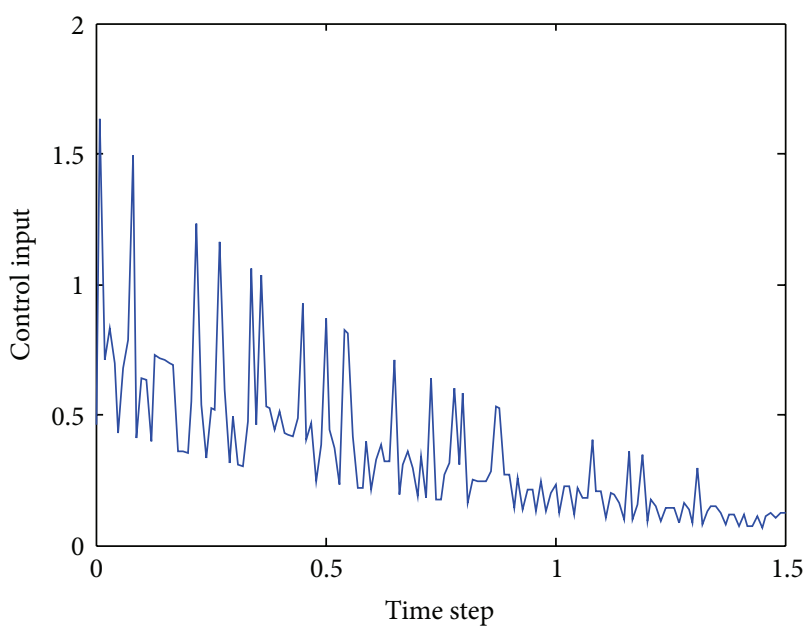

FIGURE 5: Control input under normal state feedback control.

feedback control to system (1), separately. Then, we get the simulation results in Figures 2-5.

Obviously, the unstable system (1) becomes more easily stable by using MPC strategy.

\section{Conclusions}

In this paper, MPC strategy is presented for continuous-time singular MJSs with incomplete transition rates. The controller design problem is formulated as LMI optimization algorithm. The predictive control strategy is proved to be feasible at every sampling time and it also can guarantee the mean square admissibility of the closed-loop system.

\section{Conflict of Interests}

The authors declare that there is no conflict of interests regarding the publication of this paper.

\section{Acknowledgments}

This project was jointly supported by NSFC (61203126), NSFC (61374047), BK2012111, and 111 Project (B12018).

\section{References}

[1] L. J. Chen, Y. Leng, H. F. Guo, P. Shi, and L. Zhang, " $H_{\infty}$ control of a class of discrete-time Markov jump linear systems with piecewise-constant TPs subject to average dwell time switching," Journal of the Franklin Institute, vol. 349, no. 6, pp. 1989-2003, 2012.

[2] Q. Zhang and J.-F. Zhang, "Distributed parameter estimation over unreliable networks with Markovian switching topologies," IEEE Transactions on Automatic Control, vol. 57, no. 10, pp. 2545-2560, 2012.

[3] I. Kordonis and G. P. Papavassilopoulos, "On stability and LQ control of MJLS with a markov chain with general state space," IEEE Transactions on Automatic Control, vol. 59, no. 2, pp. 535540, 2014.

[4] M. Otero, D. H. Barmak, C. O. Dorso, H. G. Solari, and M. A. Natiello, "Modeling dengue outbreaks," Mathematical Biosciences, vol. 232, no. 2, pp. 87-95, 2011.

[5] H. Zhang, Y. Shi, and J. Wang, "On energy-to-peak filtering for nonuniformly sampled nonlinear systems: a markovian jump system approach," IEEE Transactions on Fuzzy Systems, vol. 22, no. 1, pp. 212-222, 2014.

[6] E. K. Boukas, S. Xu, and J. Lam, "On stability and stabilizability of singular stochastic systems with delays," Journal of Optimization Theory and Applications, vol. 127, no. 2, pp. 249-262, 2005.

[7] B. Chen and J. Chen, "Bifurcation and chaotic behavior of a discrete singular biological economic system," Applied Mathematics and Computation, vol. 219, no. 5, pp. 2371-2386, 2012.

[8] Y. Zhang and Q. Zhang, "Dynamical analysis of a delayed singular prey-predator economic model with stochastic fluctuations," Complexity, vol. 19, no. 5, pp. 23-29, 2014.

[9] F. Xu, Y. Wang, and X. Luo, "Soft sensor for inputs and parameters using nonlinear singular state observer in chemical processes," Chinese Journal of Chemical Engineering, vol. 21, no. 9, pp. 1038-1047, 2013.

[10] Y. Xia, E.-K. Boukas, P. Shi, and J. Zhang, "Stability and stabilization of continuous-time singular hybrid systems," Automatica, vol. 45, no. 6, pp. 1504-1509, 2009.

[11] L. Huang and X. Mao, "Stability of singular stochastic systems with Markovian switching," IEEE Transactions on Automatic Control, vol. 56, no. 2, pp. 424-429, 2011.

[12] Q. Zhu, "Stabilization of stochastically singular nonlinear jump systems with unknown parameters and continuously distributed delays," International Journal of Control, Automation and Systems, vol. 11, no. 4, pp. 683-691, 2013.

[13] E. K. Boukas, "On state feedback stabilization of singular systems with random abrupt changes," Journal of Optimization Theory and Applications, vol. 137, no. 2, pp. 335-345, 2008.

[14] E. K. Boukas, "On stability and stabilisation of continuous-time singular Markovian switching systems," IET Control Theory \& Applications, vol. 2, no. 10, pp. 884-894, 2008.

[15] S. Ma, C. Zhang, and S. Zhu, "Robust stability for discretetime uncertain singular Markov jump systems with actuator saturation," IET Control Theory \& Applications, vol. 5, no. 2, pp. 255-262, 2011. 
[16] D. Limon, J. M. Bravo, T. Alamo et al., "Robust MPC of constrained nonlinear systems based on interval arithmetic," IEE Proceedings-Control Theory and Applications, vol. 152, no. 3, pp. 325-332, 2005.

[17] A. Ulbig, S. Olaru, D. Dumur, and P. Boucher, "Explicit nonlinear predictive control for a magnetic levitation system," Asian Journal of Control, vol. 12, no. 3, pp. 434-442, 2010.

[18] J. M. Eklund, J. Sprinkle, and S. S. Sastry, "Switched and symmetric pursuit/evasion games using online model predictive control with application to autonomous aircraft," IEEE Transactions on Control Systems Technology, vol. 20, no. 3, pp. 604-620, 2012.

[19] F. A. Bayer, M. A. Müller, and F. Allgöwer, "Tube-based robust economic model predictive control," Journal of Process Control, vol. 24, no. 8, pp. 1237-1246, 2014.

[20] M. V. Kothare, V. Balakrishnan, and M. Morari, "Robust constrained model predictive control using linear matrix inequalities," Automatica, vol. 32, no. 10, pp. 1361-1379, 1996.

[21] J.-H. Park, T.-H. Kim, and T. Sugie, "Output feedback model predictive control for LPV systems based on quasi-min-max algorithm," Automatica, vol. 47, no. 9, pp. 2052-2058, 2011.

[22] H. Huang, D. F. He, and Q. X. Chen, "Quasi-min-max dynamic output feedback MPC for LPV systems," International Journal of System Control and Information Processing, vol. 1, no. 3, pp. 233-245, 2014.

[23] A. Ferramosca, D. Limon, and E. F. Camacho, "Economic MPC for a changing economic criterion for linear systems," IEEE Transactions on Automatic Control, vol. 59, no. 10, pp. 26572667, 2014.

[24] L. Zhang and E.-K. Boukas, "Stability and stabilization of Markovian jump linear systems with partly unknown transition probabilities," Automatica, vol. 45, no. 2, pp. 463-468, 2009.

[25] L. Zhang and J. Lam, "Necessary and sufficient conditions for analysis and synthesis of Markov jump linear systems with incomplete transition descriptions," IEEE Transactions on Automatic Control, vol. 55, no. 7, pp. 1695-1701, 2010.

[26] B.-C. Zheng and G.-H. Yang, "Sliding mode control for Markov jump linear uncertain systems with partly unknown transition rates," International Journal of Systems Science, vol. 45, no. 10, pp. 1999-2011, 2014.

[27] M. Shen, G. Zhang, Y. Yuan, and D. Ye, "Relaxed $H_{\infty}$ controller design for continuous markov jump system with incomplete transition probabilities," Circuits, Systems, and Signal Processing, vol. 33, no. 5, pp. 1393-1410, 2014.

[28] M. Shen, G. Zhang, and Y. Yuan, " $H_{\infty}$ static output feedback controller design for continuous Markov jump systems with incomplete transition probabilities," Transactions of the Institute of Measurement and Control, vol. 36, no. 6, pp. 743-753, 2014.

[29] Z. Wan and M. V. Kothare, "An efficient off-line formulation of robust model predictive control using linear matrix inequalities," Automatica, vol. 39, no. 5, pp. 837-846, 2003.

[30] L. Xie, "Output feedback $H_{\infty}$ control of systems with parameter uncertainty," International Journal of Control, vol. 63, no. 4, pp. 741-750, 1996.

[31] R. A. Horn and C. R. Johnson, Matrix Analysis, Cambridge University Press, Cambridge, UK, 1985. 

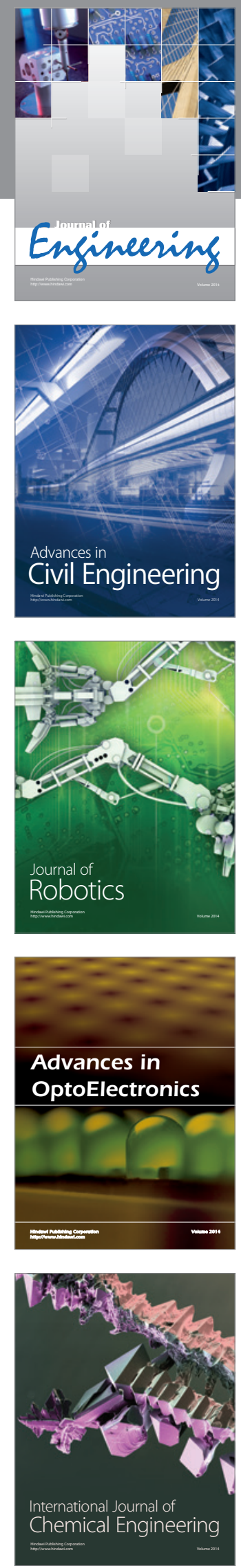

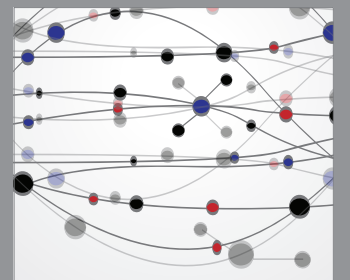

The Scientific World Journal
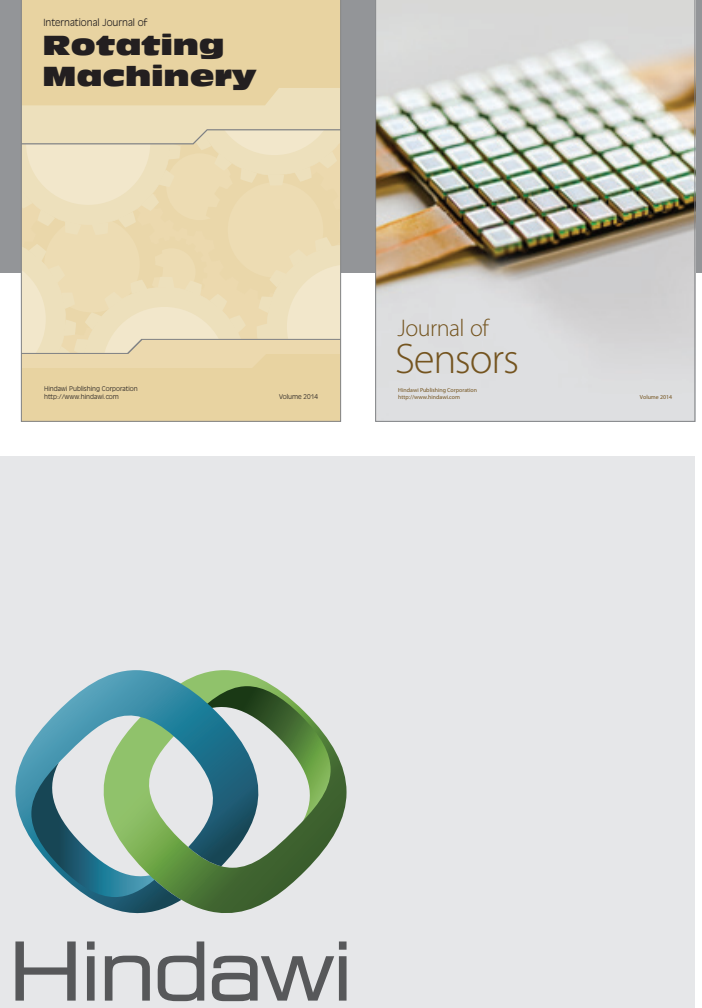

Submit your manuscripts at http://www.hindawi.com
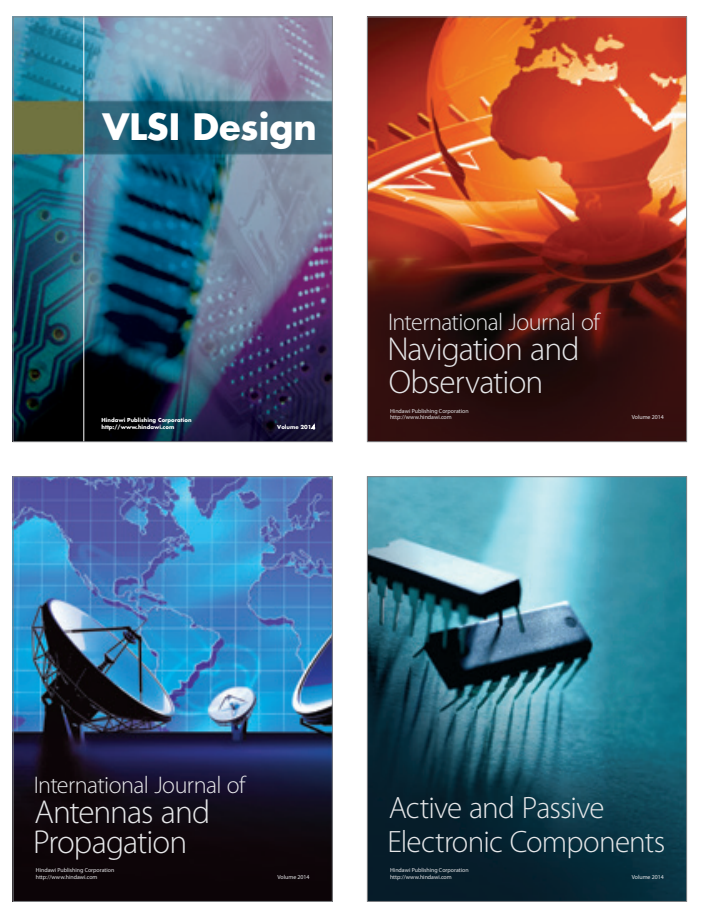
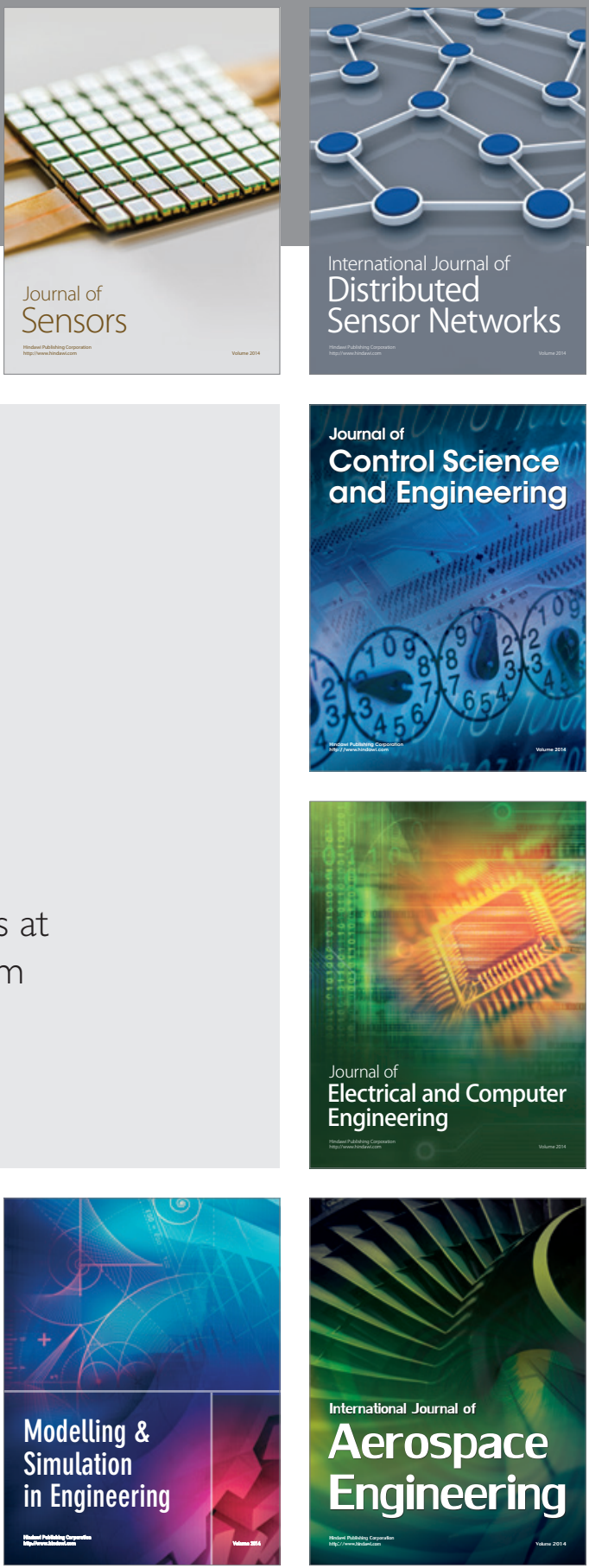

Journal of

Control Science

and Engineering
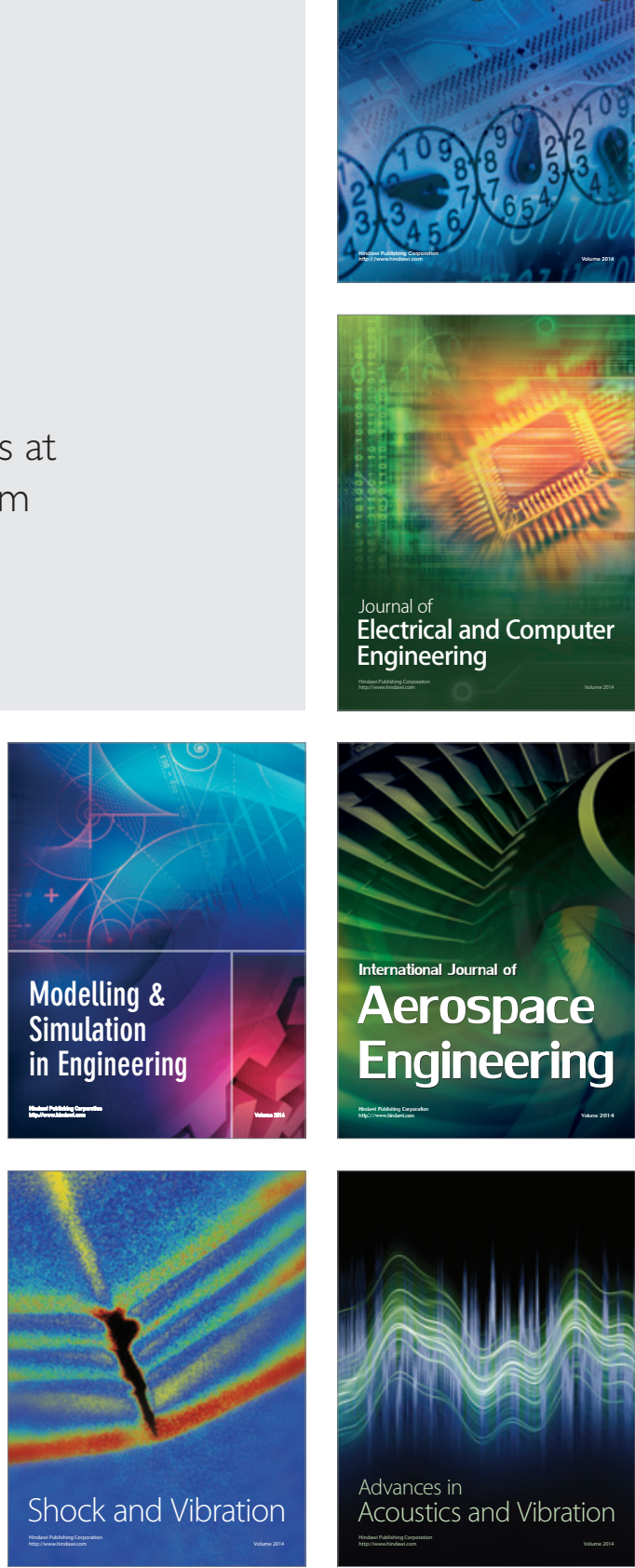